\title{
Straightforward synthesis of 1-alkyl-2-(trifluoromethyl)aziridines starting from 1,1,1-trifluoroacetone
}

\author{
Sara Kenis, ${ }^{1}$ Matthias D'hooghe,${ }^{1}{ }^{*}$ Guido Verniest, ${ }^{1, \ddagger}$ Vinh Duc Nguyen, ${ }^{2}$ Tuyet Anh Dang Thi, ${ }^{2}$ \\ Tuyen Van Nguyen, ${ }^{2}$ and Norbert De Kimpe ${ }^{1, *}$ \\ ${ }^{1}$ Department of Sustainable Organic Chemistry and Technology, Faculty of Bioscience Engineering, \\ Ghent University, Coupure Links 653, B-9000 Ghent, Belgium \\ ${ }^{2}$ Institute of Chemistry, Vietnam Academy of Science \& Technology, \\ 18 Hoang Quoc Viet, Cau Giay, Hanoi, Vietnam
}

\begin{abstract}
An efficient and straightforward approach towards the synthesis of 1-alkyl-2-(trifluoromethyl)aziridines starting from 1,1,1-trifluoroacetone via imination, $\alpha$-chlorination, hydride reduction and ring closure, was developed. In addition, novel primary $\beta$-iodo amines were obtained by regioselective ring opening of these 2-(trifluoromethyl)aziridines using alkyl iodides, and their synthetic potential was demonstrated by converting them into novel $\alpha-\mathrm{CF}_{3}-\beta$-phenylethylamines upon treatment with lithium diphenylcuprate.
\end{abstract}

\section{Introduction}

In the past decade, organofluorine compounds have gained a lot of interest in organic and medicinal chemistry. ${ }^{1}$ This is due to the fact that the incorporation of one or more fluorine atoms, for example as a trifluoromethyl group, often results in an improvement of the biological properties of bioactive compounds. ${ }^{2}$ For this reason, fluorinated and trifluoromethylated compounds are increasingly used in the pharmaceutical and agrochemical industry. ${ }^{3}$ The direct introduction of a fluorine atom or a trifluoromethyl group on heterocyclic compounds, however, is often problematic, ${ }^{4}$ and therefore a building block approach can provide a useful alternative in some cases.

Aziridines are known to be excellent building blocks for the preparation of a large variety of nitrogencontaining compounds, and numerous synthetic approaches towards the preparation of aziridines have been described. ${ }^{5}$ An interesting subclass of these three-membered rings is the class of 2 (trifluoromethyl)aziridines, and their preparation has been relatively well studied. ${ }^{6}$ However,

\footnotetext{
${ }^{*}$ matthias.dhooghe@UGent.be

${ }^{\ddagger}$ Present address: Vrije Universiteit Brussel, Faculty of Sciences and Bio-engineering Sciences, Department of Chemistry, Pleinlaan 2, B-1050 Brussel, Belgium.

*norbert.dekimpe@UGent.be
} 
although the reactivity of non-activated aziridines is known to be different and often complementary to that of their activated counterparts, ${ }^{7}$ only a few approaches towards the synthesis of nonactivated 2-(trifluoromethyl)aziridines have been reported in the literature. ${ }^{\text {hh-k }}$ In a first approach, 1alkyl-2-(trifluoromethyl)aziridines have been obtained by ring closure of $\mathrm{N}$-alkylamino alcohols using dichlorotriphenylphosphorane. ${ }^{6 \mathrm{~h}, 6 i}$ These $\mathrm{N}$-alkylamino alcohols were synthesized through ring opening of (S)-3,3,3-trifluoropropylene oxide by the corresponding alkylamines. A drawback of this procedure involves the high cost of the optically pure starting material, and the preparation of racemic 3,3,3-trifluoropropylene oxide elongates this synthetic procedure substantially. ${ }^{8}$ In a second approach, 1-alkyl-2-(trifluoromethyl)aziridines have been prepared by the reaction of ( $\beta$ trifluoromethyl)vinyl diphenyl sulfonium triflate with different alkylamines. ${ }^{6 j}$ This ( $\beta$ trifluoromethyl)vinyl diphenyl sulfonium triflate was synthesized starting from 3,3,3-trifluoro-2bromopropene, which was converted by an addition-elimination reaction with thiophenol into phenyl $\beta$-(trifluoromethyl)vinyl sulfide. ${ }^{9}$ Treatment of this sulfide with the expensive diphenyliodonium triflate results in ( $\beta$-trifluoromethyl)vinyl diphenyl sulfonium triflate. The relatively high cost of diphenyliodonium triflate, however, represents an important drawback if large-scale preparations are desired. In a third approach, dimethylsulfonium methylide has been used to synthesize 1-(2-methoxy-1-phenylethyl)-2-(trifluoromethyl)aziridine. ${ }^{6 k}$ Despite the synthetic value of the above-described procedures, their application for large-scale approaches is less attractive from an economical point of view, hence the interest in short and straightforward alternatives. Therefore, a new and convenient approach towards 1-alkyl-2-(trifluoromethyl)aziridines is disclosed in this paper, starting from the commercially available and inexpensive 1,1,1-trifluoroacetone, known as a useful building block for fluorinated compounds. ${ }^{10}$

In addition, ring-opening reactions of these 1-alkyl-2-(trifluoromethyl)aziridines were evaluated in order to provide a new and selective approach towards $\mathrm{CF}_{3}$-substituted nitrogen-containing target compounds. A number of ring-opening reactions of non-activated 2-(trifluoromethyl)aziridines have previously been studied, ${ }^{11}$ involving proton-catalyzed ring-opening reactions and aziridinium ion formation through $\mathrm{N}$-alkylation followed by ring opening. Complementary to these results, the regioselective ring opening by acetic acid and alkyl iodides was studied in this work, leading in the case of alkyl iodides to interesting novel primary $\beta$-iodo amines which can be used as precursors for the synthesis of a variety of compounds. As an example, new trifluorinated amphetamine derivatives were prepared through further elaboration of these $\beta$-iodo amines. Amphetamines are of pharmacological interest due to their stimulating or inhibiting effect on the central nervous system, their anti-inflammatory activity and their ability to inhibit several enzymes. ${ }^{12}$ Although a few methods for the preparation of $\alpha-\mathrm{CF}_{3}-\beta$-phenylethylamines are known in the literature, i.e. through 
reductive amination of 3-phenyl-1,1,1-trifluoropropan-2-one, ${ }^{13}$ the addition of Grignard reagents to fluoral hemiacetal ${ }^{14}$ and trifluoromethylation of enamines, ${ }^{15}$ this particular methodology represents a new approach in that respect.

\section{Results and discussion}

$N$-(1-Methyl-2,2,2-trifluoroethylidene)alkylamines 2 were prepared through condensation of 1,1,1trifluoroacetone 1 with 3 equiv. of the corresponding alkylamines in diethyl ether in the presence of 0.5 equiv. of titanium(IV) chloride. ${ }^{16}$ Subsequently, imines 2 were $\alpha$-chlorinated using 1 equiv. of $N$ chlorosuccinimide (NCS) in cyclohexane upon reflux for 3 hours. Traditionally, carcinogenic carbon tetrachloride is used as a solvent for this type of transformations, ${ }^{17}$ but could be replaced by cyclohexane in this case without altering the rate or yield of the reaction. Next to $\mathrm{N}$-(1-chloromethyl2,2,2-trifluoroethylidene)alkylamines 3 , the presence of the corresponding enamines ${ }^{18}$ was observed as well (ratio: 9/1). Due to their hydrolytic instability, no attempts were made to obtain analytically pure samples of $\alpha, \alpha, \alpha$-trifluoromethyl imines $\mathbf{2}$ and $\mathbf{3}$ through silicagel purification. However these imines $\mathbf{2}$ and $\mathbf{3}$ were obtained in a purity of at least $95 \%$. In the following step, $\mathrm{N}$-(1-chloromethyl2,2,2-trifluoroethylidene)alkylamines 3 were reduced by using 4 equiv. of sodium borohydride in methanol under reflux, yielding the corresponding $\beta$-chloro amines 4 in good overall yields (Scheme 1, Table 1). The spectral data obtained for compound 4 a were in full accordance with those reported in the literature. ${ }^{11 a}$ Attempted reductive cyclization of $\alpha$-chloro imines 3 towards the desired 2(trifluoromethyl)aziridines $\mathbf{5}$ using different reducing agents (e.g. 1 equiv. of $\mathrm{LiAlH}_{4}$ in $\mathrm{THF}$ at $0^{\circ} \mathrm{C}$ or 1 equiv. of $\mathrm{LiBH}_{4}$ in THF under reflux) did not lead to the desired conversion due to the reduced nucleophilicity of the nitrogen atom in amines $\mathbf{4}$, caused by the strong electron-withdrawing effect of the trifluoromethyl substituent in $\alpha$-position. Therefore, a strong base, such as lithium bis(trimethylsilyl)amide (LiHMDS), was shown to be necessary to affect ring closure of $\beta$-chloro amines 4 . In this way, several new 1-alkyl-2-(trifluoromethyl)aziridines 5 were obtained in high yields by treatment of $\alpha-\mathrm{CF}_{3}-\beta$-chloro amines 4 with 1.1 equiv. of LiHMDS in THF for 4 hours at room temperature (Scheme 1, Table 1). The spectral data obtained for compounds $\mathbf{5 a}$ and $\mathbf{5 e}$ were in full accordance with those reported in the literature, ${ }^{9,11 a}$ whereas aziridines $\mathbf{5 b}$-d have not been described before. Compared to known procedures for the preparation of 1-alkyl-2(trifluoromethyl)aziridines, which either need expensive reagents or consist of multiple reaction steps, this short and efficient approach is based on the use of commercially available and relatively inexpensive resources and is therefore a suitable alternative for large-scale applications. 


\section{Scheme 1.}

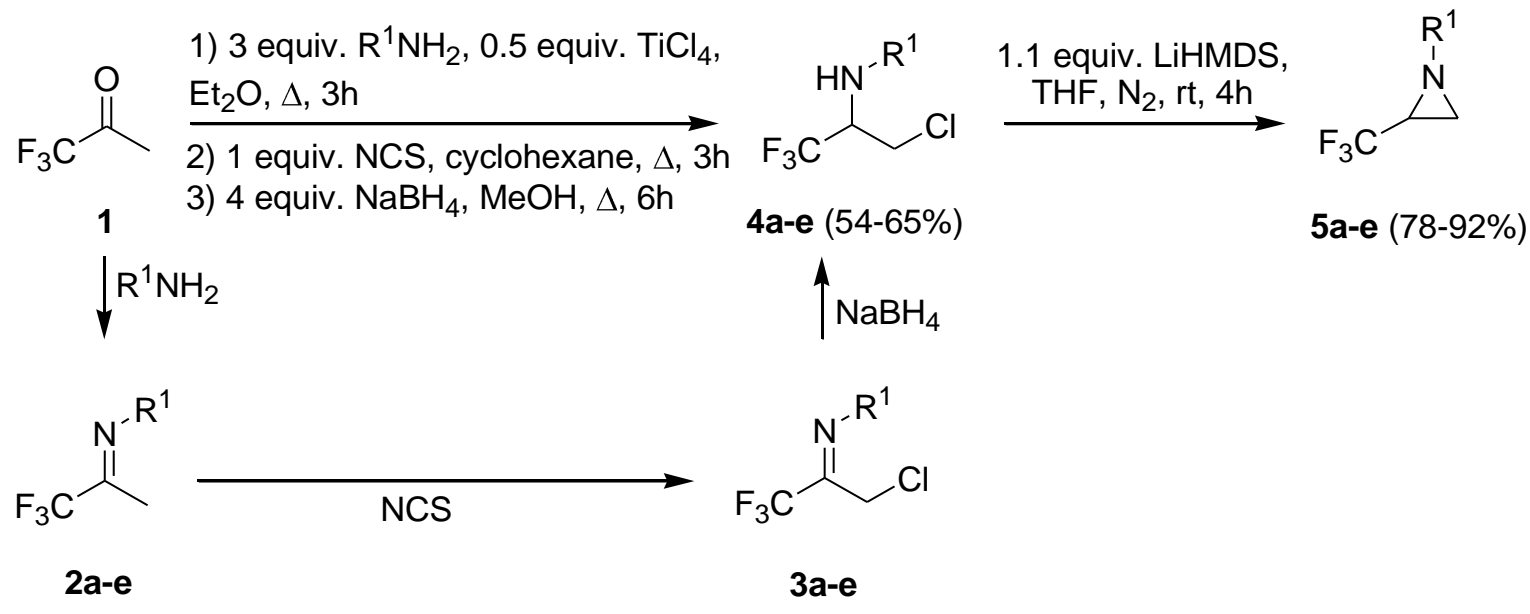

Table 1. Synthesis of $\mathrm{N}$-alkyl-3-chloro-1,1,1-trifluoropropan-2-amines 4a-e and 1-alkyl-2(trifluoromethyl)aziridines 5a-e

\begin{tabular}{ccc}
\hline Compound & $\mathbf{R}^{1}$ & Yield $^{\mathbf{a}}$ \\
\hline $\mathbf{4 a}$ & $\mathrm{Bn}$ & $56 \%$ \\
$\mathbf{4 b}$ & $4-\mathrm{ClC}_{6} \mathrm{H}_{4} \mathrm{CH}_{2}$ & $54 \%$ \\
$\mathbf{4 c}$ & $4-\mathrm{MeOC}_{6} \mathrm{H}_{4} \mathrm{CH}_{2}$ & $58 \%$ \\
$\mathbf{4 d}$ & $\mathrm{n}-\mathrm{Octyl}$ & $65 \%$ \\
$\mathbf{4 e}$ & $\left(\mathrm{CH}_{2}\right)_{2} \mathrm{C}_{6} \mathrm{H}_{5}$ & $63 \%$ \\
$\mathbf{5 a}$ & $\mathrm{Bn}$ & $92 \%$ \\
$\mathbf{5 b}$ & $4-\mathrm{ClC}_{6} \mathrm{H}_{4} \mathrm{CH}_{2}$ & $78 \%$ \\
$\mathbf{5 c}$ & $4-\mathrm{MeOC}_{6} \mathrm{H}_{4} \mathrm{CH}_{2}$ & $80 \%$ \\
$\mathbf{5 d}$ & $\mathrm{n}-\mathrm{Octyl}_{2}$ & $83 \%$ \\
$\mathbf{5 e}$ & $\left(\mathrm{CH}_{2}\right)_{2} \mathrm{C}_{6} \mathrm{H}_{5}$ & $81 \%$ \\
\hline
\end{tabular}

${ }^{a}$ Yield after column chromatography $\left(\mathrm{SiO}_{2}\right)$.

With this novel and straightforward approach towards 2-(trifluoromethyl)aziridines $\mathbf{5}$ in hand, additional studies on the reactivity of these interesting building blocks were performed. As mentioned in the introduction, Katagiri and Karimova had already explored the reactivity of nonactivated 2-(trifluoromethyl)aziridines towards a number of electrophiles and nucleophiles. ${ }^{11}$ Complementary to these studies, ring-opening reactions with acetic acid and alkyl iodides were investigated in this work. The ring opening of 1-benzyl-2-(trifluoromethyl)aziridine $\mathbf{5 a}$ to 2 benzylamino-3,3,3-trifluoropropyl acetate 6 using 5 equiv. of acetic acid in $\mathrm{CH}_{2} \mathrm{Cl}_{2}$ proceeded very sluggishly, as heating for 7 days at $60^{\circ} \mathrm{C}$ in a pressure vial was required to drive the reaction to completion, affording acetate 6 in 52\% yield. Performing the reaction in acetic acid as a solvent appeared to be less successful, as it led to more formation of impurities. The ring opening with acetic acid had already briefly been explored in the literature, resulting in the conclusion that the acidity of 
acetic acid is too weak to induce ring opening of 1-alkyl-2-(trifluoromethyl)aziridines, even after prolonged storage at $20^{\circ} \mathrm{C}^{11 \mathrm{~b}}$ The rather drastic conditions required for the acetic acid-induced ring opening of aziridine $\mathbf{5}$ a to acetate $\mathbf{6}$ (Scheme 2 ) corroborate the previously described finding that the trifluoromethyl-substituted aziridine ring is quite inert towards electrophiles due to the strong electron-withdrawing effect of the $\mathrm{CF}_{3}$-group, ${ }^{11}$ but refute the conclusion that acetic acid is too weak to affect a ring-opening reaction.

In a second part, ring opening of 1-alkyl-2-(trifluoromethyl)aziridines 5a-c with alkyl iodides towards $\beta$-iodo amines was investigated, as this approach has not been studied before (only chloride- and bromide-induced ring openings have been studied). At first, ring opening using 1 equiv. of benzyl bromide in acetonitrile was evaluated, which did not lead to complete conversion, even after 13 days of heating at reflux temperature. Attempts to accelerate the reaction, for example by replacing acetonitrile with a high-boiling solvent such as dimethylformamide and performing the reaction in acetonitrile under microwave irradiation, only resulted in complex mixtures. Finally, complete conversion was attained by treatment of 2-(trifluoromethyl)aziridines 5a-c with 1 equiv. of benzyl iodide $^{19}$ at $100^{\circ} \mathrm{C}$ under neat conditions, affording 1,1,1-trifluoro-3-iodopropan-2-amines 7a-c (Scheme 2, Table 2, Entries 1-3) in good yields. Detailed spectroscopic analysis ruled out the formation of the other regioisomers. ${ }^{11 a, 20}$ It should be noted that the ring opening of non-activated aziridines by benzyl bromide in acetonitrile constitutes a highly efficient regio- and stereoselective approach towards the preparation of secondary $\beta$-bromo amines. ${ }^{21}$ The observations made here once again confirm the reduced reactivity of $\mathrm{CF}_{3}$-substituted 1-alkylaziridines with respect to electrophiles. In addition to benzyl iodide, ring opening of 2-(trifluoromethyl)aziridines $5 \mathbf{a}-\mathbf{b}$ by methyl iodide towards 1,1,1-trifluoro-3-iodopropan-2-amines 7d-e (Scheme 2, Table 2, Entries 4-5) was attained in good yields by using 1 equiv. of methyl iodide in acetonitrile at $100^{\circ} \mathrm{C}$ for 3 days in a pressure vial (Scheme 2 Table 2). In an effort to improve the reaction rate, the amount of methyl iodide was increased to 5 equiv., which unfortunately resulted in the formation of benzyltrimethylammonium iodide as a side-product. It should be mentioned that $\beta$-iodo amines $7 a-e$ represent a novel class of compounds with unexplored synthetic potential.

As know from different literature reports, the ring opening of non-activated 2-alkylaziridines by alkyl iodides usually produces secondary $\beta$-iodo amines through ring opening of intermediate aziridinium salts at the substituted aziridine carbon atom under thermodynamic control. ${ }^{22}$ In this case, however, ring opening of the aziridinium salts $\mathbf{1 0}$ by iodide proceeded regiospecifically towards primary $\beta$-iodo amines 7 , pointing to the conclusion that this transformation probably occurs under kinetic control. This change in regioselectivity can again be attributed to the strong electron-withdrawing property of 
the $\mathrm{CF}_{3}$-substituent, which prevents thermodynamic equilibration through recyclization of primary $\beta$ iodo amines $\mathbf{7}$ towards intermediate aziridinium salts $\mathbf{1 0}$.

In order to demonstrate the synthetic potential of these novel 1,1,1-trifluoro-3-iodopropan-2-amines 7, their coupling with benzene towards novel $\beta$-phenylethylamines, an interesting class of amphetamine derivatives, was evaluated. In a first approach, $\beta$-iodo amines 7a-b were treated with phenylmagnesium chloride under various reaction conditions (2 equiv. of $\mathrm{PhMgCl}$ in $\mathrm{THF}$ or $\mathrm{CH}_{2} \mathrm{Cl}_{2}$ at $-78^{\circ} \mathrm{C}, 0^{\circ} \mathrm{C}$, room temperature or under reflux). Unfortunately, no desired $\beta$-phenylethylamines were formed, and even at low temperatures $\left(-78^{\circ} \mathrm{C}\right)$ elimination products were observed. Another attempt involved a Negishi cross-coupling of $\beta$-iodo amines 7a-b with iodobenzene. In accordance to literature procedures, ${ }^{23} \beta$-iodo amines $7 \mathbf{a}-\mathbf{b}$ were converted into the corresponding organozinc reagents using zinc, activated with catalytic iodine, in DMF. Subsequent Pd-catalysed cross-coupling of the zinc reagents with iodobenzene using $2.5 \mathrm{~mol} \%$ of $\mathrm{Pd}_{2}(\mathrm{dba})_{3}$ and $10 \mathrm{~mol} \%$ of a precatalyst such as $\mathrm{P}(\mathrm{o} \text {-tol })_{3}$ or SPhos, only resulted in complex mixtures. Finally, a successful synthesis of $\alpha-$ trifluoromethyl- $\beta$-phenylethylamines $\mathbf{8} \mathbf{a}-\mathbf{b}$ was accomplished by using a Gilman reagent such as lithium diphenylcuprate. ${ }^{24}$ Thus, $\beta$-iodo amines $7 \mathbf{a}$-b were treated with 3 equiv. of lithium diphenylcuprate in diethyl ether at $-78^{\circ} \mathrm{C}$, and stirring at room temperature for 5 hours eventually afforded the desired 1,1,1-trifluoro-3-phenylpropan-2-amines 8a-b (Scheme 2), which were purified by means of column chromatography on silica gel. In this way, 1,1,1-trifluoro-3-iodopropan-2-amines 7a-b were applied for the formation of new trifluorinated amphetamine derivatives 8a-b, demonstrating their synthetic potential. From a medicinal point of view, the introduction of an electron-withdrawing $\mathrm{CF}_{3}$ group onto a $\beta$-phenylethylamino moiety can have a pronounced influence on the basicity of the nitrogen atom and hence on the pharmacological properties of these compounds. It should be mentioned that attempted direct conversion of aziridines $\mathbf{5}$ into the corresponding $\beta$-phenylethylamines upon treatment with 1.5 equiv. PhLi in THF or with 2 equiv. $\mathrm{Ph}_{2} \mathrm{CuLi}$ in $\mathrm{Et}_{2} \mathrm{O}$ was unsuccessful, resulting in full recovery of the starting material. 


\section{Scheme 2.}

$$
\text { (52\%) }
$$

Table 2. Synthesis of 2-benzylamino-3,3,3-trifluoropropyl acetate 6 and $\mathbf{N , N}$-dialkyl-1,1,1-trifluoro3-iodopropan-2-amines 7

\begin{tabular}{ccccc}
\hline Entry & $\mathbf{R}^{\mathbf{1}}$ & $\mathbf{R}^{\mathbf{2}}$ & Reaction conditions $^{\text {a }}$ & Product (yield) $^{\text {Pa }}$ \\
\hline $\mathbf{1}$ & $\mathrm{Bn}$ & $\mathrm{Bn}$ & $\mathbf{A}$ & $\mathbf{7 a}(74 \%)$ \\
$\mathbf{2}$ & $4-\mathrm{ClC}_{6} \mathrm{H}_{4} \mathrm{CH}_{2}$ & $\mathrm{Bn}$ & $\mathbf{A}$ & $\mathbf{7 b}(88 \%)$ \\
$\mathbf{3}$ & $4-\mathrm{MeOC}_{6} \mathrm{H}_{4} \mathrm{CH}_{2}$ & $\mathrm{Bn}$ & $\mathbf{A}$ & $\mathbf{7 c}(81 \%)$ \\
$\mathbf{4}$ & $\mathrm{Bn}$ & $\mathrm{Me}$ & $\mathbf{B}$ & $\mathbf{7 d}(87 \%)$ \\
$\mathbf{5}$ & $4-\mathrm{ClC}_{6} \mathrm{H}_{4} \mathrm{CH}_{2}$ & $\mathrm{Me}$ & $\mathbf{B}$ & $\mathbf{7 e ~ ( 7 2 \% )}$ \\
\hline
\end{tabular}

${ }^{\mathrm{a}} \mathbf{A}=1$ equiv. $\mathrm{Bnl}$, neat, $100^{\circ} \mathrm{C}, 24 \mathrm{~h} ; \mathbf{B}=1$ equiv. $\mathrm{Mel}, \mathrm{CH}_{3} \mathrm{CN}, 100^{\circ} \mathrm{C}, 3 \mathrm{~d}$, pressure vial.

In conclusion, a novel and convenient method for the preparation of 1-alkyl-2(trifluoromethyl)aziridines was developed using easily accessible and inexpensive resources, making it an economical approach for large-scale synthesis. Furthermore, additional information concerning the reactivity of these 2-(trifluoromethyl)aziridines was acquired by treatment of the latter with acetic acid and alkyl iodides. In the case of alkyl iodides, novel primary $\beta$-iodo amines were obtained through regioselective ring opening of intermediate aziridinium salts, which proved to be valuable precursors for the synthesis of novel $\alpha$-trifluoromethyl- $\beta$-phenylethylamines, an interesting class of trifluorinated amphetamines derivatives. 


\section{Experimental part}

${ }^{1} \mathrm{H}$ NMR spectra were recorded at $300 \mathrm{MHz}$ (JEOL ECLIPSE+) with $\mathrm{CDCl}_{3}$ as solvent and tetramethylsilane as internal standard. ${ }^{13} \mathrm{C}$ NMR spectra were recorded at $75 \mathrm{MHz}$ (JEOL ECLIPSE+) with $\mathrm{CDCl}_{3}$ as solvent and tetramethylsilane as internal standard. ${ }^{19} \mathrm{~F}$ NMR spectra were recorded at $282 \mathrm{MHz}$ (JEOL ECLIPSE+) with $\mathrm{CDCl}_{3}$ as solvent and $\mathrm{CFCl}_{3}$ as internal standard. Electron spray (ES) mass spectra were obtained with an Agilent 1100 Series MS (ES, 4000V) mass spectrometer. Electron impact (EI) mass spectra were recorded by using a HP 6890 GC coupled to a HP 5973 MSD (mass selective detector). IR spectra were measured with a Spectrum One FT-IR spectrophotometer. High resolution electron spray (ES) mass spectra were obtained with an Agilent Technologies 6210 Series Time-of-Flight. Diethyl ether and tetrahydrofuran were dried over sodium benzophenone ketyl. Other solvents were used as received from the supplier. Melting points of crystalline compounds were measured with a Büchi 540 apparatus.

Synthesis of $\mathrm{N}$-alkyl-3-chloro-1,1,1-trifluoropropan-2-amines 4 via $\mathrm{N}$-(1-chloromethyl-2,2,2trifluoroethylidene)alkylamines 3

General procedure: To an ice-cooled solution of 1,1,1-trifluoroacetone (0.09 mol, 1 equiv.) and the alkylamine ( $0.27 \mathrm{~mol}, 3$ equiv.) in dry $\mathrm{Et}_{2} \mathrm{O}(250 \mathrm{~mL})$ was added dropwise a solution of $\mathrm{TiCl}_{4}(0.05 \mathrm{~mol}$, 0.5 equiv.) in dry petroleum ether $(80 \mathrm{~mL})$. After stirring for 3 hours under reflux, the reaction mixture was filtered over a pad of Celite ${ }^{\circledR}$ and washed with $\mathrm{Et}_{2} \mathrm{O}(2 \times 50 \mathrm{~mL})$. Evaporation of the solvent under reduced pressure afforded $\mathrm{N}$-(1-methyl-2,2,2-trifluoroethylidene)alkylamine $\mathbf{2}$ (purity $>95 \%$ based on NMR). Subsequently, a solution of imine $2(0.065 \mathrm{~mol})$ and $\mathrm{N}$-chlorosuccinimide ( 0.065 mol, 1 equiv.) in cyclohexane $(200 \mathrm{~mL})$ was heated under reflux for 3 hours. Afterwards, the resulting succinimide was filtered off and washed with cyclohexane $(2 \times 20 \mathrm{~mL})$. Evaporation of the solvent in vacuo afforded the desired $\mathrm{N}$-(1-chloromethyl-2,2,2-trifluoroethylidene)alkylamine 3 which occurs in equilibrium with a minor amount of $\mathrm{N}$-alkyl-2-amino-1-chloro-3,3,3-trifluoropropenes (ratio: 9/1). To an ice-cooled solution of the crude mixture of imine 3 (0.06 mol, 1 equiv.) in $\mathrm{MeOH}$ (150 mL) was added $\mathrm{NaBH}_{4}(0.06$ mol, 1 equiv.) in small portions whilst stirring. Subsequently, the reaction mixture was heated under reflux for 4 hours, and every hour an extra equiv. of $\mathrm{NaBH}_{4}(3$ equiv. in total) was added in small portions. Afterwards, the reaction mixture was quenched by a saturated solution of $\mathrm{NH}_{4} \mathrm{Cl}(75 \mathrm{~mL})$, extracted with EtOAc $(3 \times 50 \mathrm{~mL})$ and washed with brine $(3 \times 50$ $\mathrm{mL})$. Drying $\left(\mathrm{MgSO}_{4}\right)$, filtration of the drying agent and evaporation of the solvent afforded $\mathrm{N}$-alkyl-3chloro-1,1,1-trifluoropropan-2-amine 4 , which was purified by means of column chromatography on silicagel (hexane/EtOAc) in order to obtain an analytically pure sample. 
$N$-(1-Chloromethyl-2,2,2-trifluoroethylidene)alkylamines 3 were obtained in high purity $(>90 \%$ based on $\mathrm{NMR}, \mathrm{CDCl}_{3}$ ) and were used as such in the following step without prior purification. However, in order to confirm their structure, the spectral data of three derivatives are reported below. Despite several attempts, no conclusive mass spectra were obtained for these compounds due to their instability.

\section{$N$-(1-chloromethyl-2,2,2-trifluoroethylidene)benzylamine 3a}

Brown oil. ${ }^{1} \mathrm{H}$ NMR $\left(300 \mathrm{MHz}, \mathrm{CDCl}_{3}\right): \delta 4.18(2 \mathrm{H}, \mathrm{s}), 4.88(2 \mathrm{H}, \mathrm{d}, J=1.7 \mathrm{~Hz}), 7.30-7.40(5 \mathrm{H}, \mathrm{m}) .{ }^{13} \mathrm{C}$ $\operatorname{NMR}\left(75 \mathrm{MHz}\right.$, ref $\left.=\mathrm{CDCl}_{3}\right): \delta 30.54,55.47,119.32(q, J=278.8 \mathrm{~Hz}), 127.57,127.74,128.76,137.05$, $153.96(q, J=34.2 \mathrm{~Hz}) .{ }^{19} \mathrm{~F} \mathrm{NMR}\left(282 \mathrm{MHz}, \mathrm{CDCl}_{3}\right): \delta-72.32(3 \mathrm{~F}, \mathrm{~s}) . \mathrm{IR}\left(\mathrm{ATR}, \mathrm{cm}^{-1}\right): \mathrm{v}_{\mathrm{CN}}=1686 ; v_{\max }=$ $1454,1339,1194,1116,751,699$.

\section{$N$-(1-chloromethyl-2,2,2-trifluoroethylidene)-(4-chlorobenzyl)amine 3b}

Orange oil. ${ }^{1} \mathrm{H}$ NMR $\left(300 \mathrm{MHz}, \mathrm{CDCl}_{3}\right): \delta 4.18(2 \mathrm{H}, \mathrm{s}), 4.83(2 \mathrm{H}, \mathrm{d}, J=1.7 \mathrm{~Hz}), 7.24-7.36(4 \mathrm{H}, \mathrm{m}) .{ }^{13} \mathrm{C}$ $\operatorname{NMR}\left(75 \mathrm{MHz}\right.$, ref $\left.=\mathrm{CDCl}_{3}\right): \delta 30.48,54.54,119.32(\mathrm{q}, J=280.9 \mathrm{~Hz}), 128.74,128.96,133.23,135.52$, $154.14(\mathrm{q}, J=34.2 \mathrm{~Hz}) \cdot{ }^{19} \mathrm{~F}$ NMR $\left(282 \mathrm{MHz}, \mathrm{CDCl}_{3}\right): \delta-72.40(3 \mathrm{~F}, \mathrm{~s}) . \mathrm{IR}\left(\mathrm{ATR}, \mathrm{cm}^{-1}\right): \mathrm{v}_{\mathrm{CN}}=1636 ; v_{\max }=$ 1492, 1345, 1200, 1167, 1129, 1088, 1015, 833, 804.

\section{$N$-(1-chloromethyl-2,2,2-trifluoroethylidene)-(4-methoxybenzyl)amine 3c}

Orange oil. ${ }^{1} \mathrm{H}$ NMR $\left(300 \mathrm{MHz}, \mathrm{CDCl}_{3}\right): \delta 3.81(3 \mathrm{H}, \mathrm{s}), 4.17(2 \mathrm{H}, \mathrm{s}), 4.81(2 \mathrm{H}, \mathrm{d}, J=1.1 \mathrm{~Hz}), 6.87-6.92$ and 7.30-7.40 (4H, $2 \times \mathrm{m}) .{ }^{13} \mathrm{C} \mathrm{NMR}\left(75 \mathrm{MHz}\right.$, ref $\left.=\mathrm{CDCl}_{3}\right): \delta 30.39,54.95,55.17,114.06,119.23(\mathrm{q}, J=$ 280.4 Hz), 128.90, 128.99, 153.47 (q, $J=33.5 \mathrm{~Hz}), 158.99 .{ }^{19} \mathrm{~F} \mathrm{NMR}\left(282 \mathrm{MHz}, \mathrm{CDCl}_{3}\right): \delta-72.33(3 \mathrm{~F}, \mathrm{~s})$. IR $\left(A T R, \mathrm{~cm}^{-1}\right): \mathrm{v}_{\mathrm{CN}}=1613 ; \mathrm{v}_{\max }=1513,1247,1168,1130,1087,1033,819$.

\section{$\mathrm{N}$-alkyl-3-chloro-1,1,1-trifluoropropan-2-amines 4}

\section{3-Chloro-N-(4-chlorobenzyl)-1,1,1-trifluoropropan-2-amine 4b}

Yellow oil. $R_{\mathrm{f}}=0.28$ (Petroleum ether/ EtOAc 95/5). Yield 54\%. ${ }^{1} \mathrm{H} \mathrm{NMR}\left(300 \mathrm{MHz}, \mathrm{CDCl}_{3}\right): \delta 1.91(1 \mathrm{H}$, bs), 3.31-3.40 (1H, m), 3.63 (1H, $d \times d, J=11.8,6.6 \mathrm{~Hz}), 3.79(1 \mathrm{H}, \mathrm{d} \times \mathrm{d}, J=11.8,3.3 \mathrm{~Hz}), 3.90$ and 3.97 $(2 \mathrm{H}, 2 \times(\mathrm{d} \times \mathrm{d}), J=13.5,5.5,5.0 \mathrm{~Hz}), 7.31-7.33(4 \mathrm{H}, \mathrm{m}) .{ }^{13} \mathrm{C} \mathrm{NMR}\left(75 \mathrm{MHz}\right.$, ref $\left.=\mathrm{CDCl}_{3}\right): \delta 41.43(\mathrm{~d}, J=$ $2.3 \mathrm{~Hz}$ ), 50.82, 59.17 (q, $J=27.7 \mathrm{~Hz}$ ), 125.23 (q $J=284.6 \mathrm{~Hz}), 128.58,129.51,133.11,137.33 .{ }^{19} \mathrm{~F} \mathrm{NMR}$ $\left(282 \mathrm{MHz}, \mathrm{CDCl}_{3}\right): \delta-72.88(3 \mathrm{~F}, \mathrm{~d}, J=6.6 \mathrm{~Hz}) . \mathrm{IR}\left(\mathrm{ATR}, \mathrm{cm}^{-1}\right): \mathrm{v}_{\mathrm{NH}}=3362 ; v_{\max }=1492,1274,1162,1090$, 1015, 798, 688. MS (70 eV): $\mathrm{m} / \mathrm{z}(\%): 272 / 4\left(\mathrm{M}^{+}+1,100\right)$. HRMS (ES) calcd for $\mathrm{C}_{10} \mathrm{H}_{11} \mathrm{Cl}_{2} \mathrm{~F}_{3} \mathrm{~N}: 272.0221$ $[\mathrm{M}+\mathrm{H}]^{+}$; Found: 272.0216 . 


\section{3-Chloro-1,1,1-trifluoro-N-(4-methoxybenzyl)propan-2-amine 4c}

Yellow oil. $R_{\mathrm{f}}=0.22$ (Petroleum ether/ EtOAc 95/5). Yield 58\%. ${ }^{1} \mathrm{H} \mathrm{NMR}\left(300 \mathrm{MHz}, \mathrm{CDCl}_{3}\right): \delta 1.88(1 \mathrm{H}$, bs), 3.34-3.40 (1H, m), $3.63(1 \mathrm{H}, \mathrm{d} \times \mathrm{d}, J=11.6,6.6 \mathrm{~Hz}), 3.77(1 \mathrm{H}, \mathrm{d} \times \mathrm{d}, J=11.6,3.9 \mathrm{~Hz}), 3.81(3 \mathrm{H}, \mathrm{s})$, 3.86 and $3.94(2 \mathrm{H}, 2 \times \mathrm{d}, J=13.2 \mathrm{~Hz}), 6.86-6.91$ and 7.23-7.35 $(4 \mathrm{H}, 2 \times \mathrm{m}) .{ }^{13} \mathrm{C} \mathrm{NMR}(75 \mathrm{MHz}$, ref $=$ $\left.\mathrm{CDCl}_{3}\right): \delta 41.34\left(\mathrm{CH}_{2} \mathrm{Cl}, \mathrm{d}, J=2.3 \mathrm{~Hz}\right), 50.88,54.97,58.83(\mathrm{q}, J=27.7 \mathrm{~Hz}), 113.75,125.30(\mathrm{q}, J=283.8$ $\mathrm{Hz}), 129.37,130.75,158.92 .{ }^{19} \mathrm{~F} \mathrm{NMR}\left(282 \mathrm{MHz}, \mathrm{CDCl}_{3}\right): \delta-72.84(3 \mathrm{~F}, \mathrm{~d}, J=6.6 \mathrm{~Hz})$. IR $\left(\mathrm{ATR}, \mathrm{cm}^{-1}\right): \mathrm{v}_{\mathrm{NH}}$ $=3358 ; v_{\max }=2838,1513,1247,1165,1121,1033,830$. GC-MS (EI): $\mathrm{m} / \mathrm{z}(\%): 267$ (M+1 15), 266 (19), 236 (7), $136(7), 121(100)$.

\section{$N$-(3-Chloro-1,1,1-trifluoropropan-2-yl)octan-1-amine 4d}

Yellow oil. $R_{\mathrm{f}}=0.26$ (Petroleum ether/ EtOAc 99/1). Yield 65\%. ${ }^{1} \mathrm{H}$ NMR (300 MHz, $\left.\mathrm{CDCl}_{3}\right): \delta$ 0.86-0.90 $(2 \mathrm{H}, \mathrm{m}), 0.88(3 \mathrm{H}, \mathrm{t}, J=6.8 \mathrm{~Hz}), 1.23-1.35(8 \mathrm{H}, \mathrm{m}), 1.45-1.56(2 \mathrm{H}, \mathrm{m}), 2.67-2.81(2 \mathrm{H}, \mathrm{m}), 3.27-3.38(1 \mathrm{H}$, $\mathrm{m}), 3.61(1 \mathrm{H}, \mathrm{d} \times \mathrm{d}, J=11.6,6.6 \mathrm{~Hz}), 3.79(1 \mathrm{H}, \mathrm{d} \times \mathrm{d}, J=11.6,3.3 \mathrm{~Hz}) .{ }^{13} \mathrm{C} \mathrm{NMR}\left(75 \mathrm{MHz}, \mathrm{ref}=\mathrm{CDCl}_{3}\right): \delta$ 13.96, 22.62, 26.94, 29.21, 29.36, 30.16, 31.79, 41.42 (d, $J=3.5 \mathrm{~Hz}), 48.21,60.73$ (q, $J=27.7 \mathrm{~Hz}$ ), $125.26(\mathrm{q}, J=284.4 \mathrm{~Hz}) \cdot{ }^{19} \mathrm{~F}$ NMR $\left(282 \mathrm{MHz}, \mathrm{CDCl}_{3}\right): \delta-73.34(3 \mathrm{~F}, \mathrm{~d}, J=6.6 \mathrm{~Hz}) . \operatorname{IR}\left(\mathrm{ATR}, \mathrm{cm}^{-1}\right): v_{\mathrm{NH}}=$ $3320 ; v_{\max }=1467,1270,1148,1120,688 . G C-M S(E I): m / z(\%): 259\left(M^{+}, 1\right), 160$ (100).

\section{3-Chloro-1,1,1-trifluoro-N-(2-phenylethyl)propan-2-amine 4e}

Yellow oil. $R_{\mathrm{f}}=0.36$ (Petroleum ether/EtOAc 95/5). Yield 63\%. ${ }^{1} \mathrm{H}$ NMR $\left(300 \mathrm{MHz}, \mathrm{CDCl}_{3}\right): \delta$ 2.75-2.88 $(2 \mathrm{H}, \mathrm{m}), 2.95-3.07$ and 3.17-3.24 (2H, $2 \times \mathrm{m}), 3.33-3.41(1 \mathrm{H}, \mathrm{m}), 3.57(1 \mathrm{H}, \mathrm{d} \times \mathrm{d}, J=11.6,7.2 \mathrm{~Hz}), 3.76$ $(1 \mathrm{H}, \mathrm{d} \times \mathrm{d}, J=11.6,3.9 \mathrm{~Hz}) \cdot 7.19-7.33(5 \mathrm{H}, \mathrm{m}) \cdot{ }^{13} \mathrm{C} \mathrm{NMR}\left(75 \mathrm{MHz}\right.$, ref $\left.=\mathrm{CDCl}_{3}\right): \delta 36.46,41.26(\mathrm{~d}, J=$ $2.3 \mathrm{~Hz}$ ), 49.24, 60.62 (q, $J=27.7 \mathrm{~Hz}), 125.14$ (q $J=283.8 \mathrm{~Hz}), 126.25,128.41,128.58,139.06 .{ }^{19} \mathrm{~F} \mathrm{NMR}$ $\left(282 \mathrm{MHz}, \mathrm{CDCl}_{3}\right): \delta-73.30(3 \mathrm{~F}, \mathrm{~d}, J=6.6 \mathrm{~Hz}) . \mathrm{IR}\left(\mathrm{ATR}, \mathrm{cm}^{-1}\right): v_{\mathrm{NH}}=3350 ; v_{\max }=1454,1266,1123,1087$, 749, 698. GC-MS (EI): m/z (\%): 251 (M+1 0.1), 216 (1), 160 (100).

\section{Synthesis of 1-alkyl-2-(trifluoromethyl)aziridines 5}

General procedure: In a flame-dried flask, $\mathrm{N}$-alkyl-3-chloro-1,1,1-trifluoropropan-2-amine 4 (0.05 mol, 1 equiv.) was dissolved in dry THF $(100 \mathrm{~mL})$ under nitrogen atmosphere. The resulting mixture was then cooled to $0^{\circ} \mathrm{C}$ and LiHMDS (1.1 equiv., $1 \mathrm{M}$ in THF) was added dropwise via a syringe. After stirring at room temperature for 4 hours, the reaction mixture was quenched with a saturated solution of $\mathrm{NH}_{4} \mathrm{Cl}(50 \mathrm{~mL})$, extracted with EtOAc $(3 \times 25 \mathrm{~mL})$ and washed with brine $(3 \times 25 \mathrm{~mL})$. Drying $\left(\mathrm{MgSO}_{4}\right)$, filtration of the drying agent and evaporation of the solvent afforded 1-alkyl-2- 
(trifluoromethyl)aziridine 5, which was purified by means of column chromatography on silicagel (hexane/EtOAc) to obtain an analytically pure sample.

\section{1-(4-Chlorobenzyl)-2-(trifluoromethyl)aziridine 5b}

Yellow oil. $R_{\mathrm{f}}=0.28$ (Petroleum ether/EtOAc 95/5). Yield 83\%. ${ }^{1} \mathrm{H} \mathrm{NMR}\left(300 \mathrm{MHz}, \mathrm{CDCl}_{3}\right): \delta 1.65(1 \mathrm{H}$, $\mathrm{d}, J=6.1 \mathrm{~Hz}), 2.09-2.17(2 \mathrm{H}, \mathrm{m}), 3.46$ and $3.53(2 \mathrm{H}, 2 \times \mathrm{d}, J=13.8 \mathrm{~Hz}), 7.25-7.32(4 \mathrm{H}, \mathrm{m}) .{ }^{13} \mathrm{C} \mathrm{NMR}(75$ $\mathrm{MHz}$, ref $\left.=\mathrm{CDCl}_{3}\right): \delta 30.04,37.40(\mathrm{q}, J=39.2 \mathrm{~Hz}), 62.65,123.98(\mathrm{q}, J=272.3 \mathrm{~Hz}), 128.59,129.24$, 133.27, 135.92. ${ }^{19} \mathrm{~F} \mathrm{NMR}\left(282 \mathrm{MHz}, \mathrm{CDCl}_{3}\right.$, ref $\left.=\mathrm{CFCl}_{3}\right): \delta-70.93(3 \mathrm{~F}, \mathrm{~d}, J=5.3 \mathrm{~Hz}) . \mathrm{IR}\left(\mathrm{ATR}, \mathrm{cm}^{-1}\right): v_{\max }=$ 1492, 1284, 1138, 1088, 1016, 803. MS (70 eV): m/z (\%): 236/8 ( $\left.{ }^{+}+1,100\right)$. HRMS (ES) calcd for $\mathrm{C}_{10} \mathrm{H}_{10} \mathrm{ClF}_{3} \mathrm{~N}: 236.0454[\mathrm{M}+\mathrm{H}]^{+}$; Found: 236.0451.

\section{1-(4-Methoxybenzyl)-2-(trifluoromethyl)aziridine 5c}

Yellow oil. $R_{\mathrm{f}}=0.25$ (petroleum ether/EtOAc 95/5). Yield 80\%. ${ }^{1} \mathrm{H} \mathrm{NMR}\left(300 \mathrm{MHz}, \mathrm{CDCl}_{3}\right): \delta 1.63(1 \mathrm{H}$, $\mathrm{d}, J=5.5 \mathrm{~Hz}), 2.07-2.15(2 \mathrm{H}, \mathrm{m}), 3.44$ and $3.50(2 \mathrm{H}, 2 \times \mathrm{d}, J=13.8 \mathrm{~Hz}), 3.78(3 \mathrm{H}, \mathrm{s}), 6.85-6.89$ and 7.21-7.26 (4H, $2 \times \mathrm{m}) .{ }^{13} \mathrm{C} \mathrm{NMR}\left(75 \mathrm{MHz}, \mathrm{CDCl}_{3}\right): \delta 29.83,37.19$ (q, $\left.J=29.2 \mathrm{~Hz}\right), 55.24,62.83,113.93$, $124.24(q, J=272.3 \mathrm{~Hz}), 129.32,129.46,159.16 .{ }^{19} \mathrm{~F} \mathrm{NMR}\left(282 \mathrm{MHz}, \mathrm{CDCl}_{3}\right.$, ref $\left.=\mathrm{CFCl}_{3}\right): \delta-71.52(3 \mathrm{~F}$, $\mathrm{d}, J=4.0 \mathrm{~Hz}$ ). IR (ATR, $\left.\mathrm{cm}^{-1}\right): v_{\max }=1613,1513,1284,1243,1135,1032,811 . \mathrm{MS}(70 \mathrm{eV}): \mathrm{m} / \mathrm{z}(\%): 232$ $\left(\mathrm{M}^{+}+1,100\right)$. HRMS (ES) calcd for $\mathrm{C}_{11} \mathrm{H}_{13} \mathrm{~F}_{3} \mathrm{NO}: 232.0949[\mathrm{M}+\mathrm{H}]^{+}$; Found: 232.0953.

\section{1-Octyl-2-(trifluoromethyl)aziridine 5d}

Light-yellow oil. $R_{\mathrm{f}}=0.29$ (Petroleum ether/EtOAc 98/2). Yield 78\%. ${ }^{1} \mathrm{H}$ NMR $\left(300 \mathrm{MHz}, \mathrm{CDCl}_{3}\right): \delta 0.85-$ $0.90(2 \mathrm{H}, \mathrm{m}), 0.88(3 \mathrm{H}, \mathrm{t}, J=6.3 \mathrm{~Hz}), 1.23-1.39(8 \mathrm{H}, \mathrm{m}), 1.50(1 \mathrm{H}, \mathrm{d}, J=6.6 \mathrm{~Hz}), 1.54-1.61(2 \mathrm{H}, \mathrm{m})$, 1.91-1.99 $(1 \mathrm{H}, \mathrm{m}), 2.07(1 \mathrm{H}, \mathrm{d}, J=3.3 \mathrm{~Hz}), 2.16-2.25$ and 2.38-2.47 $(2 \mathrm{H}, 2 \times \mathrm{m}) .{ }^{13} \mathrm{C}$ NMR $(75 \mathrm{MHz}$, ref $\left.=\mathrm{CDCl}_{3}\right): \delta 14.05,22.62,27.06,29.15,29.33,29.44,30.04(\mathrm{~d}, J=2.3 \mathrm{~Hz}), 31.78,37.32(\mathrm{q}, J=39.2 \mathrm{~Hz}$ ), 60.59, $124.21(\mathrm{q}, J=272.3 \mathrm{~Hz}) .{ }^{19} \mathrm{~F} \mathrm{NMR}\left(282 \mathrm{MHz}, \mathrm{CDCl}_{3}\right): \delta-71.16(3 \mathrm{~F}, \mathrm{~d}, J=5.3 \mathrm{~Hz})$. IR $\left(\right.$ ATR, $\left.\mathrm{cm}^{-1}\right)$ : $v_{\max }=1405,1286,1155,1089,700,658 . \mathrm{GC}-\mathrm{MS}(\mathrm{EI}): \mathrm{m} / \mathrm{z}(\%): 223\left(\mathrm{M}^{+}, 0.54\right) ;\left(\mathrm{M}^{+}-\mathrm{CH}_{2} \mathrm{CH}_{3}, 8\right) ;\left(\mathrm{M}^{+}-\right.$ $\left.\left.\left(\mathrm{CH}_{2}\right)_{2} \mathrm{CH}_{3}, 26\right) ;\left(\mathrm{M}^{+}-\left(\mathrm{CH}_{2}\right)_{4} \mathrm{CH}_{3}, 34\right) ;\left(\mathrm{M}^{+}-\mathrm{CH}_{2}\right)_{6} \mathrm{CH}_{3}, 100\right)$. HRMS (ES) calcd for $\mathrm{C}_{11} \mathrm{H}_{21} \mathrm{~F}_{3} \mathrm{~N}: 224.1626$ $[\mathrm{M}+\mathrm{H}]^{+}$; Found: 224.1626 .

\section{Synthesis of 2-benzylamino-3,3,3-trifluoropropyl acetate 6}

In a $20 \mathrm{~mL}$ pressure vial, 1-alkyl-2-(trifluoromethyl)aziridine 5 a $\left(0.5 \mathrm{mmol}, 1\right.$ equiv.) and $\mathrm{CH}_{3} \mathrm{COOH}$ (5 mmol, 5 equiv.) were dissolved in $\mathrm{CH}_{2} \mathrm{Cl}_{2}(3 \mathrm{~mL})$, after which the reaction mixture was stirred for 7 days at $60^{\circ} \mathrm{C}$. Afterwards, the reaction mixture was neutralised by means of a saturated solution of 
$\mathrm{NaHCO}_{3}$, poured into water $(4 \mathrm{~mL})$ and extracted with $\mathrm{CH}_{2} \mathrm{Cl}_{2}(3 \times 2 \mathrm{~mL})$. The combined organic layers were dried over anhydrous magnesium sulfate. Filtration of the drying agent and evaporation of the solvent afforded 2-benzylamino-3,3,3-trifluoropropyl acetate 6, which was purified by means of column chromatography on silicagel (hexane/EtOAc) to obtain an analytically pure sample ( $25 \%$ yield, $33 \mathrm{mg})$.

Light-yellow oil. $R_{\mathrm{f}}=0.21$ (Petroleum ether/EtOAc 90/10). Yield 25\%. ${ }^{1} \mathrm{H}$ NMR $\left(300 \mathrm{MHz}, \mathrm{CDCl}_{3}\right.$ ): $\delta$ $1.71(1 \mathrm{H}, \mathrm{bs}), 2.06(3 \mathrm{H}, \mathrm{s}), 3.29-3.39(1 \mathrm{H}, \mathrm{m}), 3.90$ and $4.01(4 \mathrm{H}, 2 \times \mathrm{d}, J=13.2 \mathrm{~Hz}), 4.22(1 \mathrm{H}, \mathrm{d} \times \mathrm{d}, J=$ $12.1 \mathrm{~Hz}, 4.4 \mathrm{~Hz}), 4.29(1 \mathrm{H}, \mathrm{d} \times \mathrm{d}, J=12.1 \mathrm{~Hz}, 5.5 \mathrm{~Hz}), 7.26-7.35(5 \mathrm{H}, \mathrm{m}) .{ }^{13} \mathrm{C} \mathrm{NMR}\left(75 \mathrm{MHz}, \mathrm{CDCl}_{3}\right): \delta$ 20.70, 51.66, $57.27(q, J=27.7 \mathrm{~Hz}), 61.12\left(\mathrm{CH}_{2} \mathrm{O}\right), 125.70(\mathrm{q}, J=283.8 \mathrm{~Hz}), 127.47,128.20,128.55$, 139.06, 170.57. ${ }^{19} \mathrm{~F} \mathrm{NMR}\left(282 \mathrm{MHz}, \mathrm{CDCl}_{3}\right): \delta-73.00(3 \mathrm{~F}, \mathrm{~d}, J=6.6 \mathrm{~Hz}) . \mathrm{IR}\left(\mathrm{ATR}, \mathrm{cm}^{-1}\right): \mathrm{v}_{\mathrm{NH}}=3359 ; \mathrm{v}_{\mathrm{CO}}=$ 1745; $v_{\max }=1226,1133,1047,739,698$. MS (70 eV): $\mathrm{m} / \mathrm{z}(\%): 262\left(\mathrm{M}^{+}+1,100\right)$. HRMS (ES) calcd for $\mathrm{C}_{12} \mathrm{H}_{15} \mathrm{~F}_{3} \mathrm{NO}_{2}: 262.1055[\mathrm{M}+\mathrm{H}]^{+}$; Found: 262.1047.

\section{Synthesis of $N$-alkyl- $N$-benzyl-1,1,1-trifluoro-3-iodopropan-2-amines 7a-c}

General procedure: A mixture of 1-alkyl-2-(trifluoromethyl)aziridine 5 ( $6 \mathrm{mmol}, 1$ equiv.) and benzyl iodide ${ }^{19}$ ( 6 mmol, 1 equiv.) was heated at $100^{\circ} \mathrm{C}$ under neat conditions for $24 \mathrm{~h}$, affording $\beta$-iodo amine 7 in $72-88 \%$ yield. This compound was purified by recrystallisation from absolute EtOH or by means of column chromatography on silicagel (hexane) to obtain an analytically pure sample.

\section{N,N-Dibenzyl-1,1,1-trifluoro-3-iodopropan-2-amine 7a}

White crystals. Recrystallisation from absolute EtOH; $\mathrm{Mp}=94.8^{\circ} \mathrm{C}$. Yield $32 \% .{ }^{1} \mathrm{H} \mathrm{NMR}(300 \mathrm{MHz}$, $\left.\mathrm{CDCl}_{3}\right): \delta$ 3.30-3.46 $(3 \mathrm{H}, \mathrm{m}), 3.72$ and $3.99(4 \mathrm{H}, 2 \times \mathrm{d}, J=13.2 \mathrm{~Hz}), 7.25-7.37$ and 7.44-7.47 $(10 \mathrm{H}, \mathrm{m})$. ${ }^{13} \mathrm{C} \mathrm{NMR}\left(75 \mathrm{MHz}, \mathrm{CDCl}_{3}\right): \delta-2.92,53.96,60.43$ (q, $J=25.4 \mathrm{~Hz}$ ), 125.56 (q, $J=294.2 \mathrm{~Hz}$ ), 127.52, 128.36 and 129.45, 137.79. ${ }^{19} \mathrm{~F} \mathrm{NMR}\left(282 \mathrm{MHz}, \mathrm{CDCl}_{3}\right): \delta-67.14(3 \mathrm{~F}, \mathrm{~d}, J=7.9 \mathrm{~Hz}) . \mathrm{IR}\left(\mathrm{ATR}, \mathrm{cm}^{-1}\right): v_{\max }$ $=1238,1144,1109,1090,748,697 . \mathrm{MS}(70 \mathrm{eV}): \mathrm{m} / \mathrm{z}(\%): 420\left(\mathrm{M}^{+}+1,100\right)$. HRMS (ES) calcd for $\mathrm{C}_{17} \mathrm{H}_{18} \mathrm{~F}_{3} \mathrm{IN}: 420.0436[\mathrm{M}+\mathrm{H}]^{+}$; Found: 420.0414.

\section{$N$-Benzyl-N-(4-chlorobenzyl)-1,1,1-trifluoro-3-iodopropan-2-amine 7b}

White crystals. Recrystallisation from absolute $\mathrm{EtOH} ; \mathrm{Mp}=70.3^{\circ} \mathrm{C}$. Yield $50 \% .{ }^{1} \mathrm{H} \mathrm{NMR}(300 \mathrm{MHz}$, $\left.\mathrm{CDCl}_{3}\right): \delta 3.26-3.45(3 \mathrm{H}, \mathrm{m}), 3.70$ and $3.95(2 \mathrm{H}, 2 \times \mathrm{d}, J=13.2 \mathrm{~Hz}), 3.70$ and $3.97(2 \mathrm{H}, 2 \times \mathrm{d}, J=13.8$ $\mathrm{Hz}$ ), 7-27-7.44 and 7.44-7.47 (9H, m). ${ }^{13} \mathrm{C} \mathrm{NMR}\left(75 \mathrm{MHz}, \mathrm{CDCl}_{3}\right): \delta-3.00,53.35,54.02,60.58$ (q, $J=$ $25.4 \mathrm{~Hz}$ ), 125.44 (q, $J=293.1 \mathrm{~Hz}$ ), 127.64, 128.42, 128.53, 129.46, 130.76, 133.28, 136.34, 137.55. ${ }^{19} \mathrm{~F}$ $\operatorname{NMR}\left(282 \mathrm{MHz}, \mathrm{CDCl}_{3}\right.$, ref $\left.=\mathrm{CFCl}_{3}\right): \delta-67.88(3 \mathrm{~F}, \mathrm{~d}, J=7.9 \mathrm{~Hz}) . \mathrm{IR}\left(\mathrm{ATR}, \mathrm{cm}^{-1}\right): v_{\max }=2852,1490,1260$, 
1235, 1164, 1144, 1088, 1100, 1074, 695. MS (70 eV): m/z (\%): 454/6 $\left(\mathrm{M}^{+}+1,100\right)$. HRMS (ES) calcd for $\mathrm{C}_{17} \mathrm{H}_{17} \mathrm{ClF}_{3} \mathrm{IN}: 454.0046[\mathrm{M}+\mathrm{H}]^{+}$; Found: 454.0055.

N-Benzyl-1,1,1-trifluoro-3-iodo-N-(4-methoxybenzyl)propan-2-amine 7c

Light-yellow oil. $R_{\mathrm{f}}=0.29$ (Petroleum ether/EtOAc 98/2). Yield 34\%. ${ }^{1} \mathrm{H} \mathrm{NMR}\left(300 \mathrm{MHz}, \mathrm{CDCl}_{3}\right): \delta 3.30-$ $3.45(3 \mathrm{H}, \mathrm{m}), 3.81(3 \mathrm{H}, \mathrm{s}), 3.65$ and $3.98(2 \mathrm{H}, 2 \times \mathrm{d}, J=13.2 \mathrm{~Hz}), 3.69$ and $3.92(2 \mathrm{H}, 2 \times \mathrm{d}, J=13.8 \mathrm{~Hz})$, 6.86-6.89, 7-25-7.36 and 7.44-7.47 (9H, $3 \times \mathrm{m}) .{ }^{13} \mathrm{C} \mathrm{NMR}\left(75 \mathrm{MHz}, \mathrm{CDCl}_{3}\right): \delta-2.72,53.38,53.67,55.22$, $60.28(q, J=25.4 \mathrm{~Hz}), 113.70,125.56(q, J=293.1 \mathrm{~Hz}$ ), 127.46, 128.36, 129.38, 129.67, 130.69, 138.02, 159.02. ${ }^{19} \mathrm{~F} \mathrm{NMR}\left(282 \mathrm{MHz}, \mathrm{CDCl}_{3}\right.$, ref $\left.=\mathrm{CFCl}_{3}\right): \delta-67.76(3 \mathrm{~F}, \mathrm{~d}, J=7.9 \mathrm{~Hz})$. IR $\left(\mathrm{ATR}, \mathrm{cm}^{-1}\right): v_{\max }=$ $1511,1241,1164,1143,1105,1036,738,698$. MS (70 eV): $\mathrm{m} / \mathrm{z}(\%): 450\left(\mathrm{M}^{+}+1,100\right)$. HRMS (ES) calcd for $\mathrm{C}_{18} \mathrm{H}_{20} \mathrm{~F}_{3}$ INO: $450.0542[\mathrm{M}+\mathrm{H}]^{+}$; Found: 450.0539.

\section{Synthesis of $\mathrm{N}$-alkyl-1,1,1-trifluoro-3-iodo- $\mathrm{N}$-methylpropan-2-amines $7 \mathrm{~d}$-e}

General procedure: In a $20 \mathrm{~mL}$ pressure vial, 1-alkyl-2-(trifluoromethyl)aziridine 5 (2 mmol, 1 equiv.) and Mel ( $2 \mathrm{mmol}, 1$ equiv.) were dissolved in acetonitrile $(6 \mathrm{~mL})$. After stirring for 3 days at $100^{\circ} \mathrm{C}$, the solvent was removed in vacuo, affording $N$-alkyl-1,1,1-trifluoro-3-iodo- $N$-methylpropan-2-amine 7, which was purified by means of column chromatography on silicagel (hexane) to obtain an analytically pure sample.

\section{N-Benzyl-1,1,1-trifluoro-3-iodo- $N$-methylpropan-2-amine 7d}

Light-yellow oil. $R_{\mathrm{f}}=0.34$ (Hexane). Yield 12\%. ${ }^{1} \mathrm{H}$ NMR $\left(300 \mathrm{MHz}, \mathrm{CDCl}_{3}\right): \delta 2.35(3 \mathrm{H}, \mathrm{s}), 3.28-3.48$ $(3 \mathrm{H}, \mathrm{m}), 3.85$ and $3.96(2 \mathrm{H}, 2 \times \mathrm{d}, J=13.8 \mathrm{~Hz}), 7.24-7.46(5 \mathrm{H}, \mathrm{m}) .{ }^{13} \mathrm{C} \mathrm{NMR}\left(75 \mathrm{MHz}, \mathrm{CDCl}_{3}\right): \delta-1.72$, $35.78,59.37,66.29(q, J=25.4 \mathrm{~Hz}), 125.26$ (q, $J=295.4 \mathrm{~Hz}$ ), 127.41, 128.42, 128.86, 138.66. ${ }^{19} \mathrm{~F} \mathrm{NMR}$ $\left(282 \mathrm{MHz}, \mathrm{CDCl}_{3}\right): \delta-68.55(3 \mathrm{~F}, \mathrm{~d}, J=7.9 \mathrm{~Hz}) . \mathrm{IR}\left(\mathrm{ATR}, \mathrm{cm}^{-1}\right): v_{\max }=1712,1454,1251,1163,1105$, 1075, 734, 698. MS (70 eV): $\mathrm{m} / \mathrm{z}(\%): 344\left(\mathrm{M}^{+}+1,20\right)$. HRMS (ES) calcd for $\mathrm{C}_{11} \mathrm{H}_{14} \mathrm{~F}_{3} \mathrm{IN}: 344.0123$ $[\mathrm{M}+\mathrm{H}]^{+}$; Found: 344.0106 .

\section{$N$-(4-Chlorobenzyl)-1,1,1-trifluoro-3-iodo- $N$-methylpropan-2-amine 7e}

Yellow oil. $R_{\mathrm{f}}=0.34$ (Hexane). Yield 10\%. ${ }^{1} \mathrm{H}$ NMR $\left(300 \mathrm{MHz}, \mathrm{CDCl}_{3}\right): \delta 2.32(3 \mathrm{H}, \mathrm{s}), 3.26-3.44(3 \mathrm{H}, \mathrm{m})$, 3.81 and $3.92(2 \mathrm{H}, 2 \times \mathrm{d}, J=13.8 \mathrm{~Hz}), 7.30$ and $7.39(4 \mathrm{H}, 2 \times \mathrm{d}, J=8.3 \mathrm{~Hz}) \cdot{ }^{13} \mathrm{C} \mathrm{NMR}\left(75 \mathrm{MHz}, \mathrm{CDCl}_{3}\right): \delta$ $-1.80,35.58,58.63,66.25$ (q, $J=25.8 \mathrm{~Hz}$ ), 125.15 (q, $J=293.1 \mathrm{~Hz}), 128.51,130.04,133.01,137.09 .{ }^{19} \mathrm{~F}$ $\operatorname{NMR}\left(282 \mathrm{MHz}, \mathrm{CDCl}_{3}\right): \delta-68.01(3 \mathrm{~F}, \mathrm{~d}, J=6.6 \mathrm{~Hz}) . \mathrm{IR}\left(\mathrm{ATR}, \mathrm{cm}^{-1}\right): v_{\max }=1490,1321,1252,1163,1104$, 
1076, 1014, 837, 804. MS (70 eV): m/z (\%): 378/80 ( $\left.\mathrm{M}^{+}+1,45\right)$. HRMS (ES) calcd for $\mathrm{C}_{11} \mathrm{H}_{13} \mathrm{ClF}_{3} \mathrm{IN}$ : $377.9733[\mathrm{M}+\mathrm{H}]^{+}$; Found: 377.9724 .

\section{Synthesis of $N, N$-dialkyl-1,1,1-trifluoro-3-phenylpropan-2-amines 8}

A suspension of $\mathrm{Cul} \mathrm{(} 3 \mathrm{mmol}, 3$ equiv.) in dry $\mathrm{Et}_{2} \mathrm{O}$ was cooled to $-78^{\circ} \mathrm{C}$ and PhLi (6 equiv., $1.8 \mathrm{M}$ in dibutyl ether) was slowly added to the solution under nitrogen atmosphere via a syringe. After stirring for $30 \mathrm{~min}$ at $-78^{\circ} \mathrm{C}$, a solution of $\mathrm{N}$-benzyl-1,1,1-trifluoro-3-iodopropan-2-amine 7 (1 mmol) in dry $\mathrm{Et}_{2} \mathrm{O}$ was added at $-78^{\circ} \mathrm{C}$, and the resulting suspension was further stirred for 5 hours at room temperature. Afterwards, the reaction mixture was quenched with a saturated solution of $\mathrm{NH}_{4} \mathrm{Cl}(10$ $\mathrm{mL})$, filtered through a pad of Celite ${ }^{\circledR}$ and washed with $\mathrm{Et}_{2} \mathrm{O}(2 \times 10 \mathrm{~mL})$. The filtrate was poured into $\mathrm{H}_{2} \mathrm{O}(15 \mathrm{~mL})$, extracted with $\mathrm{Et}_{2} \mathrm{O}(3 \times 10 \mathrm{~mL})$, and the combined organic layers were washed with a saturated solution of $\mathrm{NH}_{4} \mathrm{Cl}(15 \mathrm{~mL})$ and brine $(15 \mathrm{~mL})$. Drying $\left(\mathrm{MgSO}_{4}\right)$, filtration of the drying agent and evaporation of the solvent under reduced pressure afforded $N, N$-dialkyl-1,1,1-trifluoro-3phenylpropan-2-amine $\mathbf{9}$, which was purified by means of column chromatography on silica gel (hexane) in order to obtain an analytically pure sample.

\section{$N, N$-Dibenzyl-1,1,1-trifluoro-3-phenylpropan-2-amine 8a}

Colourless oil. $R_{\mathrm{f}}=0.30$ (Hexane). Yield: $48 \% .{ }^{1} \mathrm{H}$ NMR $\left(300 \mathrm{MHz}, \mathrm{CDCl}_{3}\right): \delta 2.96-2.99(2 \mathrm{H}, \mathrm{m}), 3.42-$ $3.56(1 \mathrm{H}, \mathrm{m}), 3.73$ and $3.88(4 \mathrm{H}, 2 \times \mathrm{d}, J=13.8 \mathrm{~Hz}), 6.59-6.63(2 \mathrm{H}, \mathrm{m}), 6.96-7.48(13 \mathrm{H}, \mathrm{m}) .{ }^{13} \mathrm{C} \mathrm{NMR}$ $\left(75 \mathrm{MHz}\right.$, ref $\left.=\mathrm{CDCl}_{3}\right): \delta 32.52,53.85,59.86(\mathrm{q}, J=24.6 \mathrm{~Hz}), 126.55,127.06,127.38,127.61(\mathrm{q}, J=$ $281.9 \mathrm{~Hz}), 128.22,128.74,129.51,138.55,140.91 .{ }^{19} \mathrm{~F} \mathrm{NMR}\left(282 \mathrm{MHz}, \mathrm{CDCl}_{3}\right.$, ref $\left.=\mathrm{CFCl}_{3}\right): \delta-68.52$ $(3 F, d, J=7.9 \mathrm{~Hz})$. IR (ATR, $\left.\mathrm{cm}^{-1}\right): v_{\max }=1454,1247,1165,1140,1101,744,697 . \mathrm{MS}(70 \mathrm{eV}): \mathrm{m} / \mathrm{z}(\%):$ $370\left(\mathrm{M}^{+}+1,100\right)$. HRMS (ES) calcd for $\mathrm{C}_{23} \mathrm{H}_{23} \mathrm{~F}_{3} \mathrm{~N}$ : $370.1783[\mathrm{M}+\mathrm{H}]^{+}$; Found: 370.1779 .

\section{$N$-Benzyl-N-(4-chlorobenzyl)-1,1,1-trifluoro-3-phenylpropan-2-amine 8b}

Colourless oil. $R_{\mathrm{f}}=0.25$ (Hexane). Yield: $50 \% .{ }^{1} \mathrm{H}$ NMR $\left(300 \mathrm{MHz}, \mathrm{CDCl}_{3}\right): \delta 2.90-3.01(2 \mathrm{H}, \mathrm{m}), 3.39-$ $3.52(1 \mathrm{H}, \mathrm{m}), 3.68$ and $3.81(2 \mathrm{H}, 2 \times \mathrm{d}, J=13.8 \mathrm{~Hz}), 3.71$ and $3.87(2 \mathrm{H}, 2 \times \mathrm{d}, J=13.8 \mathrm{~Hz}), 6.93-7.41$ $(14 \mathrm{H}, \mathrm{m}) .{ }^{13} \mathrm{C} \mathrm{NMR}\left(75 \mathrm{MHz}, \mathrm{CDCl}_{3}\right): \delta 32.52,53.25,53.83,59.99$ (q, $\left.J=24.6 \mathrm{~Hz}\right), 127.24,127.58$ (q, $J=$ $291.1 \mathrm{~Hz}), 127.84,128.31,128.37,128.77,129.49,130.02,130.59,137.09,137.34,138.31 .{ }^{19} \mathrm{~F} N \mathrm{NM}$ $\left(282 \mathrm{MHz}_{\mathrm{CDCl}}\right.$, ref $\left.=\mathrm{CFCl}_{3}\right): \delta-68.62(3 \mathrm{~F}, \mathrm{~d}, J=7.9 \mathrm{~Hz})$. IR $\left(\right.$ ATR, $\left.\mathrm{cm}^{-1}\right): v_{\max }=1491,1455,1247,1141$, 1097, 747, 698. MS (70 eV): $m / z(\%): 404 / 6\left(\mathrm{M}^{+}+1,100\right)$. HRMS (ES) calcd for $\mathrm{C}_{23} \mathrm{H}_{22} \mathrm{ClF} 3 \mathrm{~N}: 404.1393$ $[\mathrm{M}+\mathrm{H}]^{+}$; Found: 404.1389 


\section{Acknowledgements}

The authors are indebted to Ghent University (GOA), the Research Foundation-Flanders (FWOVlaanderen) and the Vietnamese National Foundation for Science and Technology Development (NAFOSTED) for financial support.

\section{References}

${ }^{1}$ For reviews, see: (a) D. O'Hagan, Chem. Soc. Rev., 2008, 37, 308; (b) K. L. Kirk, J. Fluorine Chem., 2006, 127, 1013; (c) K. L. Kirk, Org. Process Res. Dev., 2008, 12, 305; (d) J. Hutchinson and G. Sandford, Curr. Chem., 1997, 193, 1; (e) J. T. Welch and S. Eswarakrishnan, Fluorine in Bioorganic Chemistry, John Wiley \& Sons: New York, 1991.

${ }^{2}$ (a) S. Purser, P. R. Moore, S. Swallow and V. Gouverneur, Chem. Soc. Rev., 2008, 37, 320; (b) W. K. Hagmann, J. Med. Chem., 2008, 51, 4359; (c) H.-J. Böhm, D. Banner, S. Bendels, M. Kansy, B. Kuhn, K. Müller, U. Obst-Sander and M. Stahl, ChemBioChem, 2004, 5, 637; (d) B. E. Smart, J. Fluorine Chem., 2001, 109, 3.

${ }^{3}$ (a) W. R. Dolbier, J. Fluorine Chem., 2005, 126, 157; (b) P. Maienfisch and R.-G Hall, Chimia, 2004, 58, 93; (c) G. Sandford, Phil. Trans. R. Soc. Lond. A, 2000, 358, 455; (d) J. T. Welch, Tetrahedron, 1987, 43, 3123.

${ }^{4}$ P. Lin and J. L. Jiang, Tetrahedron, 2000, 56, 3635.

${ }^{5}$ (a) D. Tanner, Angew. Chem. Int. Ed. Engl., 1994, 33, 599; (b) H. M. I. Osborn and J. Sweeney, Tetrahedron: Asymmetry, 1997, 8, 1693; (c) J. B. Sweeney, Chem. Soc. Rev., 2002, 31, 247; (d) X. E. Hu, Tetrahedron, 2004, 60, 2701; (e) A. Padwa and S. S. Murphree, Arkivoc, 2006, (iii), 6; (d) G. S. Singh, M. D'hooghe and N. De Kimpe, Chem. Rev., 2007, 107, 2080; (e) P. Lu, Tetrahedron, 2010, 66, 2549; (f) W. McCoull and F. A. Davis, Synthesis, 2000, 1347; (g) B. Zwanenburg and P. ten Holte in Stereoselective Hetereocyclic Chemistry III, ed. P. Metz, Springer, Berlin, 2001, 93; (h) I. D. G. Watson, L. Yu and A. K. Yudin, Acc. Chem. Res., 2006, 39, 194.

${ }^{6}$ (a) F. Grellepois, J. Nonnenmacher, F. Lachaud and C. Portella, Org. Biomol. Chem., 2011, 9, 1160; (b) S. Fioravanti, D. Colantoni, L. Pellacani and P. A. Tardella, J. Org. Chem., 2005, 70, 3296; (c) D. Colantoni, S. Fioravanti, L. Pellacani and P.A. Tardella, Org. Lett., 2004, 6, 197; (d) T. Akiyama, S. Ogi and K. Fuchibe, Tetrahedron Lett., 2003, 44, 4011; (e) K. Tanaka, M. Ohsuga, Y. Sugimoto, Y. Okafuyi and K. Mitsuhashi, J. Fluorine Chem., 1988, 39, 39; (f) V. A. Petrov, J. Fluorine Chem., 2000, 106, 25; (g) B. Crousse, S. Narizuka, D. Bonnet-Delpon and J.-P. Bégué, Synlett, 2001, 679; (h) Y. Yamauchi, T. Kawate, H. Itahashi, T. Katagiri and K. Uneyama, Tetrahedron Lett., 2003, 44, 6319; (i) T. Katagiri, H. 
Ihara, M. Takahashi, S. Kashino, K. Furuhashi and K. Uneyama, Tetrahedron: Asymmetry, 1997, 8, 2933; (j) R. Maeda, K. Ooyama, R. Anno, M. Shiosaki, T. Azema and T. Hanamoto, Org. Lett., 2010, 12, 2548; (k) K. Higashiyama, M. Matsumura, A. Shiogama, T. Yamauchi and S. Ohmiya, Heterocycles, $2002,58,85$.

${ }^{7}$ (a) J. M. Yun, T. B. Sim, H. S. Hahm, W. K. Lee and H.-J. Ha, J. Org. Chem., 2003, 68, 7675; (b) M. D'hooghe, A. Waterinckx and N. De Kimpe, J. Org. Chem., 2005, 70, 227; (c) H. J. Joon, Y.-W Kim, B. K. Lee, W. K. Lee, Y. Kimb and H.-J. Ha, Chem. Commun., 2007, 79; (d) T. Ingebrigtsen and T. Lejon, Heterocycles, 2007, 71, 891; (e) Y. Kim, H.-J. Ha, S. Y. Yun and W. K. Lee, Chem. Commun., 2008, 4363; (f) S. Catak, M. D'hooghe, T. Verstraelen, K. Hemelsoet, A. Van Nieuwenhove, H.-J. Ha, M. Waroquier, N. De Kimpe and V. Van Speybroeck, J. Org. Chem., 2010, 75, 4530; (g) K. Vervisch, M. D’hooghe, K. W. Törnroos and N. De Kimpe, J. Org. Chem., 2010, 75, 7734; (h) M. D'hooghe, S. Kenis, K. Vervisch, C. Lategan, P. J. Smith, K. Chibale and N. De Kimpe, Eur. J. Med. Chem., 2011, 46, 579.

${ }^{8}$ P.V. Ramachandran and K. J. Padiya, J. Fluorine Chem., 2007, 128, 1255.

${ }^{9}$ T. Hanamoto, R. Anno, K. Yamada, K. Ryu, R. Maeda, K. Aoi and H. Furuno, Tetrahedron, 2009, 65, 2757.

${ }^{10}$ (a) Y. Katsuhara, M. Aramaki, A. Ishii, T. Kume, C. Kawashima and S. Mitsumoto, J. Fluorine Chem., 2006, 127, 8; (b) J. A. Barten, K. Funabiki, G.-V. Röschenthaler, J. Fluorine Chem., 2002, 113, 105.

${ }^{11}$ (a) T. Katagiri, M. Takahashi, Y. Fujiwara, H. Ihara and K. Uneyama, J. Org. Chem., 1999, 64, 7323; (b) N. M. Karimova, Y. L. Teplenicheva, A.F. Kolomiets and A. V. Fokin, Russ. Chem. Bull., 1997, 46, 1136.

${ }^{12}$ (a) R. Laske, W. Meindl, E. Holler and H. Schönenberger, Arch. Pharm., 1989, 322, 847. (b) S. W. Pelletier, Chemistry of Alkaloïds, Van Nostrand-Reinhold: New York, 1970; (c) B. Testa and B. Salvesen, J. Pharm. Sci., 1980, 69, 497.

${ }^{13}$ (a) T. Ono, V.P. Kukhar and A. Vadim, J. Org. Chem., 1996, 61, 6563; (b) A. Ishii, F. Miyamoto, K. Higashiyama and K. Mikami, Chem. Lett., 1998, 119.

${ }^{14}$ (a) A. Ishii and K. Higashiyama, Synlett, 1997, 1381; (b) S. Fries, J. Pytkowicz and T. Brigaud, Tetrahedron Letters, 2005, 46, 4761.

${ }^{15}$ R. T. Gritsenko, V. V. Levin, A. D. Dilman, P. A. Belyakov, M. I. Struchkova and V. A. Tartakovsky, Tetrahedron Lett., 2009, 50, 2994.

${ }^{16}$ (a) A.V. Gulevich, N. E. Shevchenko, E. S. Balenkova, G.-V. Röschenthaler and V. G. Nenajdenko, Tetrahedron, 2008, 64, 11706; (b) J. A. Barten, E. Lork and G.-V Röschenthaler, J. Fluorine Chem., 2004, 125, 1039. 
${ }^{17}$ (a) N. De Kimpe and N. Schamp, Org. Prep. Proced. Int., 1979, 11, 115; (b) N. De Kimpe, R. Verhé, L. De Buyck and N. Schamp, J. Org. Chem., 1980, 45, 5319; (c) N. De Kimpe, E. Stanoeva, R. Verhé and N. Schamp, Synthesis, 1988, 587.

${ }^{18}$ N. De Kimpe, R. Verhé, L. De Buyck, H. Hasma and N. Schamp, Tetrahedron, 1976, 32, 3063.

${ }^{19}$ S.V. Ley, M. N. Tackett, M. L. Maddess, J. C. Anderson, P. E. Brennan, M. W. Cappi, J. P. Heer, C. Helgen, M. Kori, C. Kouklovsky, S. P. Marsden, J. Norman, D. P. Osborn, M. A. Palomero, J. B. J. Pavey, C. Pinel, L. A. Robinson, J. Schnaubelt, J. S. Scott, C. D. Spilling, H. Watanabe, K. E. Wesson and M. C. Willis, Chem. Eur. J., 2009, 15, 2874.

${ }^{20}$ A.-C. Cantet, H. Carreyre, J.-P. Gesson, B. Renoux and M.-P. Jouannetaud, Tetrahedron Letters, 2006, 47, 5547.

${ }^{21}$ (a) M. D'hooghe, W. Van Brabant and N. De Kimpe, J. Org. Chem., 2004, 69, 2703; (b) M. D'hooghe, V. Van Speybroeck, M. Waroquier and N. De Kimpe, Chem. Commun., 2006, 1554; (c) S. Y. Yun, S. Catak, W. K. Lee, M. D’hooghe, N. De Kimpe, V. Van Speybroeck, M. Waroquier, Y. Kim and H.-J. Ha, Chem. Commun, 2009, 2508.

${ }^{22}$ (a) M. D’hooghe, S. Catak, S. Stanković, M. Waroquier, Y. Kim, H.-J. Ha, V. Van Speybroeck and N. De Kimpe, Eur. J. Org. Chem., 2010, 4920; (b) L. Testa, M. Akssira, E. Zaballos-García, P. Arroyo, L.R. Domingo and J. Sepúlved-Arques, Tetrahedron, 2003, 59, 677.

${ }^{23}$ (a) A. J. Ross, H. L. Lang and R. F. W. Jackson, J. Org. Chem., 2010, 75, 245; (b) H. E. Bartrum and R. F. W. Jackson, Synlett, 2009, 2257.

${ }^{24}$ (a) H. O. House, W. L. Respess and G. M. Whitesides, J. Org. Chem., 1966, 31, 3128; (b) M. D'hooghe, M. Rottiers, R. Jolie and N. De Kimpe, Synlett, 2005, 931. 